\title{
Collaborative Visualization: Definition, Challenges, and Research Agenda
}

\author{
Petra Isenberg $^{\dagger} \quad$ Niklas Elmqvist* Jean Scholtz ${ }^{\mathbb{q}} \quad$ Daniel Cernea $^{\ddagger} \quad$ Kwan-Liu Ma ${ }^{\$} \quad$ Hans Hagen $^{\ddagger}$ \\ ${ }^{\dagger}$ INRIA *Purdue University $\quad$ "PNNL ${ }^{\ddagger}$ University of Kaiserslautern $\quad$ \$ University of California, Davis
}

\begin{abstract}
The conflux of two growing areas of technologycollaboration and visualization - into a new research direction, collaborative visualization, provides new research challenges. Technology now allows us to easily connect and collaborate with one another-in settings as diverse as over networked computers, across mobile devices, or using shared displays such as interactive walls and tabletop surfaces. Digital information is now regularly accessed by multiple people in order to share information, to view it together, to analyze it, or to form decisions. Visualizations are used to deal more effectively with large amounts of information while interactive visualizations allow users to explore the underlying data. While researchers face many challenges in collaboration and in visualization, the emergence of collaborative visualization poses additional challenges but is also an exciting opportunity to reach new audiences and applications for visualization tools and techniques.
\end{abstract}

The purpose of this article is (1) to provide a definition, clear scope, and overview of the evolving field of collaborative visualization, (2) to help pinpoint the unique focus of collaborative visualization with its specific aspects, challenges, and requirements within the intersection of general computer-supported cooperative work (CSCW) and visualization research, and (3) to draw attention to important future research questions to be addressed by the community. We conclude by discussing a research agenda for future work on collaborative visualization and urge for a new generation of visualization tools that are designed with collaboration in mind from their very inception.

Keywords: collaboration, visualization, computersupported cooperative work (CSCW), teamwork.

The final, definitive version of this article is published in Information Visualization, 10(4):310-326, October/2011 by SAGE Publications Ltd, All rights reserved. (C) [The Author(s)]

\section{Introduction}

Collaboration has been named one of the grand challenges for visualization and visual analytics, ${ }^{1}$ and for good reason: the problems that analysts face in the real world are becoming increasingly large and complex, not to mention uncertain, ill-defined, and broadly scoped. It is often no longer feasible for a single analyst to tackle the immense datasets that are now commonplace in the real world-realistic problems often require broad expertise, diverse perspectives, and a number of dedicated people to solve. In addition, interaction with digital information is increasingly becoming a social activity, for example, on the social web or on large interactive display technologies in public spaces ${ }^{2}$ and visualization research is only just beginning to expand its focus into domains outside of the work environment. ${ }^{3}$

Meanwhile, traditional visualization and visual analytics tools are typically designed for a single user interacting with a visualization application on a standard desktop computer. Extending these tools to include support for collaboration would clearly go a long way towards increasing the scope and applicability of visualization in the real world. However, the emerging field of collaborative visualization is intrinsically interdisciplinary in nature, incorporating well-established research fields such as distributed computing, human-computer interaction (HCI), and, in particular, computer-supported cooperative work (or CSCW). As an outsider to these fields, becoming familiar with their research in order to start one's own work on collaborative visualization can be a daunting task; for example, CSCW research spans 25 years and multiple conferences, journals, and textbooks that have all advanced the field through the years. ${ }^{4}$

While collaborative visualization benefits from work in other disciplines, there are many challenges, aspects, and issues that are unique to the intersection of collaborative work and visualization. These are the places where researchers have to play a significant role in expanding the state of the art and help to shape where and how visualizations will be used in the future.

In particular, CSCW research generally does not deal with data analysis challenges coupled with interactive 
The final, definitive version of this article is published in Information Visualization, 10(4):310-326, October/2011 by SAGE Publications Ltd, All rights reserved. (c) [The Author(s)]

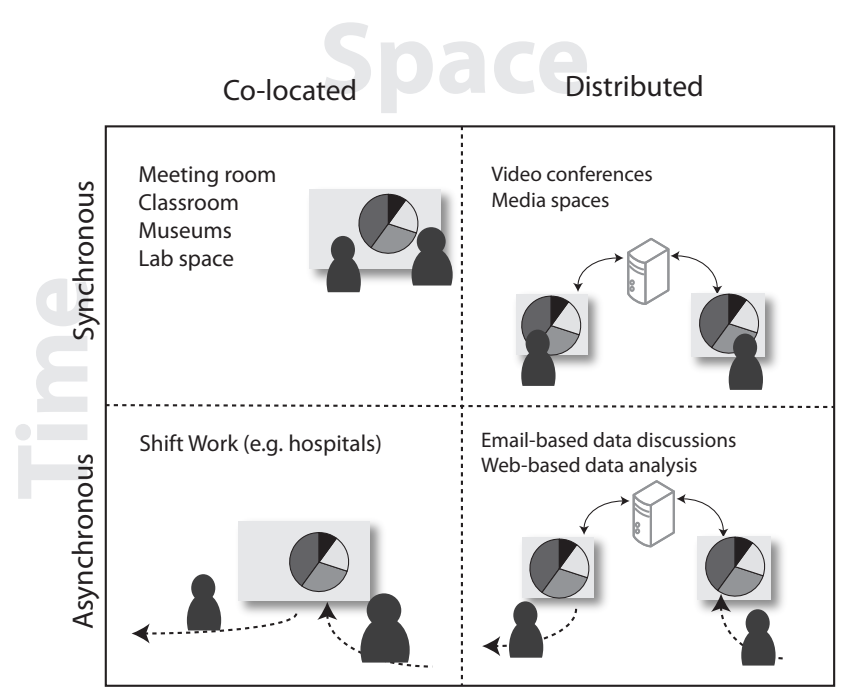

Figure 1: Collaborative visualization can occur in many scenarios delineated according to space and time. ${ }^{\text {matrix adapted from } 4,5}$

visual data representations and much work remains to be done to study collaborative data analysis, sensemaking, and perception with and of visualizations in all of the settings of the classic space-time matrix (Figure 1). Naturally, for this to be possible, visualization researchers must first arm themselves with the prerequisite knowledge, terminology, and culture that apply from the CSCW field. Only then will we be able to identify the areas where we can best contribute and apply our knowledge and expertise.

The purpose of this article is to help visualization researchers with their investigations into collaborative visualizations. It is meant to be useful for those researchers who may already have a background in collaborative visualization as well as those who are just planning their first projects. The goals of this article are (1) to provide a definition, clear scope, and overview of the evolving field of collaborative visualization, (2) to help pinpoint the unique focus of collaborative visualization with its specific aspects, challenges, and requirements within the intersection of general computersupported cooperative work (CSCW) and visualization research, and (3) to draw attention to important future research questions to be addressed by the community.

We begin by discussing a broad definition of collaborative visualization. We then study a set of representative examples of areas where collaborative visualization-as it fits our definition-has shown to be extremely beneficial to data analysis: web-based collaborative visualization, collaboration in scientific visualization, synchronous collaborative visualization for dynamic analysis environments, and collaborative analysis for environmental and mission planning. Drawing on this discussion, we propose a research agenda for future work on collaborative visualization and to usher in a new generation of information visualization tools that were designed with collaboration in mind from their very inception.

\section{Definition}

Previously, several definitions have been given to describe specific aspects of collaborative visualization. None, however, have attempted to give an encompassing definition of the entire scope of group work around visual representations of data. In the following we discuss four previous definitions, note their limitations, and finally provide our own definition for collaborative visualization.

One of the earliest definitions emphasizes the goal of collaborative visualization:

"Collaborative visualization enhances the traditional visualization by bringing together many experts so that each can contribute toward the common goal of the understanding of the object, phenomenon, or data under investigation."6

While bringing experts together is an advantage in some collaborative visualization scenarios, collaborators often do not need to be experts. Non-experts can join in collaborative analyses and learn from others' analysis processes and viewpoints on a dataset. ${ }^{7}$ Similar to this restriction by type of collaborators, other definitions may have been too restrictive in terms of the applicable fields:
"The term "collaborative visualization" refers to a subset of CSCW applications in which control over parameters or products of the scientific vi- sualization process is shared."
"Collaborative visualization [...] allows geo- graphically separated users to access a shared virtual environment to visualize and manipu- late datasets for problem solving without physi- cal travel." 9

The first definition emphasizes collaboration with interactive, manipulable visualizations for the scientific visualization community. The restriction to only the scientific visualization community is overly limiting as the information visualization and visual analytics community similarly make use of collaborative systems to analyze data. The second definition emphasizes distributed visualization in virtual environments. While much of collaborative visualization research focused on this area, e.g. 10 groupware systems have a long tradition in both distributed as well as co-located spatial domains. The limitation to virtual environments is another unnecessary restriction. Collaborative visualization also has had numerous applications outside of virtual environments.

The restriction to only interactive visualizations in both definitions may also be limiting and it is still being de- 
bated whether interactivity should be a part of a general

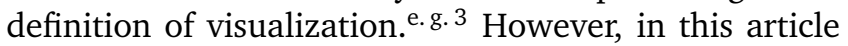
we only consider collaboration with interactive visualizations.

Recently, the term social data analysis has been coined to describe the social interaction that is a central part of collaborative visualization:

"[Social data analysis is] a version of exploratory data analysis that relies on social interaction as source of inspiration and motivation." 11

This term emphasizes the possibility of human interactions such as discussions, negotiations, or arguments around visualizations as the driving factors of data exploration. Yet, social interaction around data may occur in more scenarios than just exploratory data analysis. For example, targeted or confirmatory data analysis, teaching, learning, or decision-making scenarios around visualizations may also frequently involve collaboration.

In order to more broadly describe the entire scope that collaborative visualization can encompass, we propose to define the term collaborative visualization as follows:

Collaborative visualization is the shared use of computer-supported, (interactive,) visual representations of data by more than one person with the common goal of contribution to joint information processing activities.

This definition is derived from a general definition for visualization as the use of computer-supported, interactive, visual representations of data to amplify cognition. ${ }^{12}$ It has been augmented by emphasizing the shared use of (interactive) visual representations-which could be in the form of joint viewing, interacting with, discussing, or interpreting the representation. Secondly, the term "cognition" has been replaced with the term "information processing." This replacement acknowledges the fact that different theories exist for how cognition applies when groups come together to jointly think and reason. Each theory has different terminology, restrictions, and units of analysis. For example, the theory of Group Cognition ${ }^{13}$ describes collaborative knowledge building for small groups by focusing on linguistic analysis, Distributed Cognition ${ }^{14}$ focuses on social aspects of cognition by analyzing the coordination between individuals and artifacts, and Communities of Practice ${ }^{15}$ describe learning within much larger social communities. In order to avoid favoring any specific theory or unit of analysis, we thus use information processing as a general term to describe cognitive activities involved in individual or collaborative processing of visual information, such as reading, understanding, applying knowledge, discussing, or interpreting.

Given this broad definition of collaborative visualization, we can look at a number of different scenarios in which it may occur. Using the space-time matrix, 4,5 we can broadly categorize collaborative scenarios according to where they occur in space (distributed vs. colocated) and in time (synchronous vs. asynchronous). These distinctions for systems or tools are not strictsystems can cross boundaries and could, for example, be used both synchronously or asynchronously,e. g., rapid vs. long-term email exchanges. ${ }^{5}$ Figure 1 shows several scenarios in which collaborative visualization can occur.

Another valuable categorization for collaborative visualization systems pertains to levels of engagement teams have with a visualization system. The larger group involved in social interaction around data, for example, can simply view the information, actively interact with and explore it, or even join in creating new visualizations and share those and the underlying datasets with a larger community. ${ }^{16}$ Several digital systems have been designed to support collaborative visualizations along these different levels of engagement, as outlined below:

Viewing: Presentation systems such as PowerPoint or simple videoconferencing tools can support a group of people viewing static or animated visualizations of data without being able to interact with or annotate the information. Such scenarios often occur, for example, in classrooms or meetings where one presenter explains, teaches, or summarizes information for the larger group. The goal of the group may be to learn, discuss, interpret, or form decisions from a pre-selected set of information and visualizations.

Interacting/Exploring: When groups of people share the same interactive visualization software, either in co-located or distributed settings, they can choose and select alternative views of the data for its exploration, analysis, discussion, and interpretation. In distributed settings, findings can typically be exchanged through chat, comments, e-mail, or a video-/audio-link so that the changing views and alternative representations of the data can be discussed and analyzed. This discussion can also occur face-to-face in co-located settings. The goal of the group with this level of engagement is often to cover and explore different and more aspects of the data, consider alternative interpretations, and discuss the data in a wider visual context.

Sharing/Creating: Through the emerging trend of usergenerated content sites for visualization (e.g., in systems such as Many Eyes ${ }^{17}$ ), many people are able to create, upload, and share new datasets and visualizations. Often this type of sharing is done within a greater community to raise awareness about a certain issue.

Similar to the space-time matrix, levels of engagement do not provide a clear-cut categorization of collabora- 

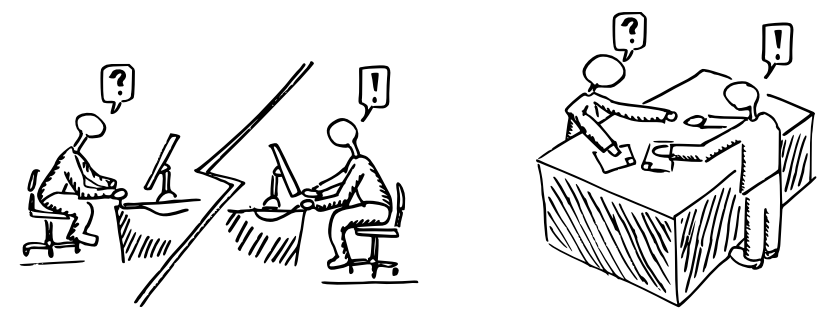

Figure 2: Distributed ${ }^{18}$ and co-located ${ }^{19}$ collaborative sensemaking. 20

tive visualization systems. Digital systems may, for example, be intended to mainly support collaborative interaction and exploration of data but may also support the sharing and creation of new visualizations or even the download of new datasets to visualize. However, both time and space dimensions as well as levels of engagement can help to broadly scope a research focus within collaborative visualization.

\section{Research Background}

Research on collaboration started in the area of scientific visualization and, thus, many early tools focused on scientific datasets and techniques (e. g., using volume or flow analysis) and distributed synchronous collaboration in specific environments such as CAVEs or using head-mounted displays.

This past focus is, for example, visible if one looks at the publications on collaborative visualization in the IEEE VisWeek conferences (Vis, InfoVis, and VAST). These three particular conferences were chosen as the top venues representing research interests of the larger visualization community but of course publications of collaborative visualization systems are also found elsewhere (ACM ITS, ACM CHI, and others).

Out of 1583 papers published in the three IEEE VisWeek conferences-VIS since 1990, InfoVis since 1995, VAST since 2006-34 papers focused on collaborative visualization and only nine covered co-located collaboration. Yet, in the past several years the support of collaborative visualization has become increasingly important as can be seen from the temporal trend in Figure 3.

The following sections briefly outline two major research streams according to their main type of spatial collaboration scenario: distributed and co-located.

\subsection{Distributed Visualization}

Within the area of distributed collaborative visualization (left image in Figure 2), one research focus has been on architectures and synchronization mechanisms for allowing efficient synchronous remote work with large scientific datasets. e.g. $9,21-23^{\text {Much of this research }}$ is focused on applications in virtual reality (VR) over the web, ${ }^{\text {e. g. } 21}$ in GRID computing, ${ }^{\text {e.g. } 24,25}$ or for special hardware environments such as CAVEs. ${ }^{\text {see }} 26$ Grimstead et al. ${ }^{18}$ provide an excellent overview and taxonomy of 42 different distributed collaborative visualization approaches which describes and characterizes this stream of research in more detail.

During the past several years, distributed web-based information visualization applications have emerged with a focus on making information visualization accessible to an internet-sized (mostly lay) audience. e. g. 17,27 With these systems, the research focus has shifted from the more technical aspects of network latency, synchronization, and view updates to more social, human-centered questions such as how wide audiences can be engaged to discuss and explore information, how laypeople can effectively share data and visualizations online, or how collaborative contributions can be effectively structured and integrated into a shared visualization to ignite further discussion and common ground formation. ${ }^{7}$

\subsection{Co-located Visualization}

Several other approaches have focused on the support of synchronous co-located collaboration with technology (right image in Figure 2). These approaches can be broadly categorized as those using single-display ${ }^{28}$ or multi-display technology.

Single-display technology often comes in the form of large interactive walls. ${ }^{\text {e. } 29}$ or tabletop displays. e. g. 30 Research in this area has, for example, described mechanisms to support coordination of activities in the workspace, e.g. 31-33 awareness of group member's activities, ${ }^{\text {e. } .34}$ or access to and transfer of items in the workspace. .e. g. 35 With emerging display technologies such as multi-touch tabletop or wall displays, independent input for each group member becomes easier and cheaper to achieve without specific hardware devices. However, additional synchronous inputs lead to new challenges. Past research ${ }^{36-38}$ has specifically addressed how people can coordinate synchronous input over visualization spaces. Two specific overview articles 7,39 provide additional detail on the applicability of CSCW research on co-located collaboration to colocated collaborative information visualization.

Research on multi-display environments is concerned with coordinating input and output from a number of different display devices, such as large displays as well as integrated mobile and wireless devices. e. g. 40 Examples of past research endeavors include those for molecular visualization across large displays and a tabletop ${ }^{41}$, for geospatial visualization across a similar setup ${ }^{42}$, or for a network setup which allows researchers to connect and share their own visualizations from laptops on large displays. ${ }^{43}$ 
The final, definitive version of this article is published in Information Visualization, 10(4):310-326, October/2011 by SAGE Publications Ltd, All rights reserved. (c) [The Author(s)]

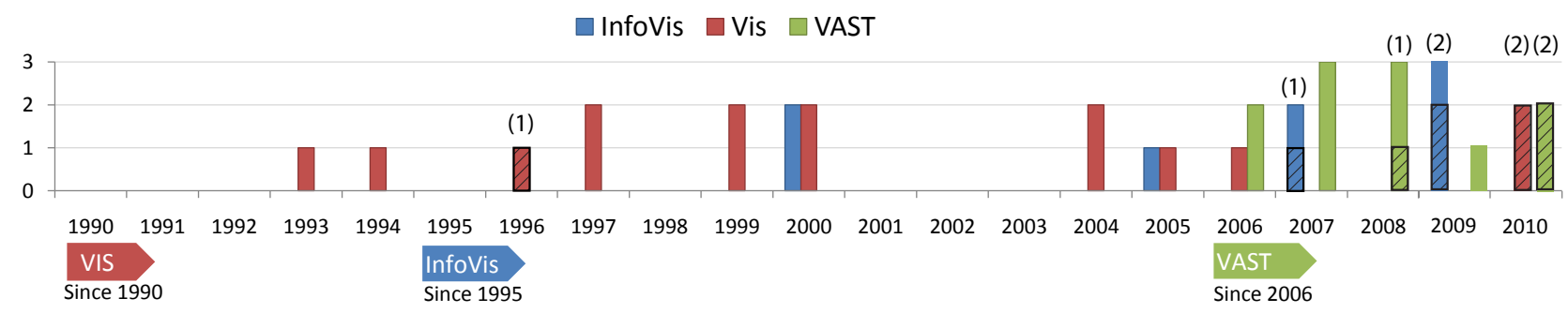

Figure 3: Papers published with a focus on collaborative visualization in three major visualization venues (IEEE Vis, InfoVis, and VAST). Shading and numbers above a bar indicate the number of papers on co-located collaboration for a venue per year.

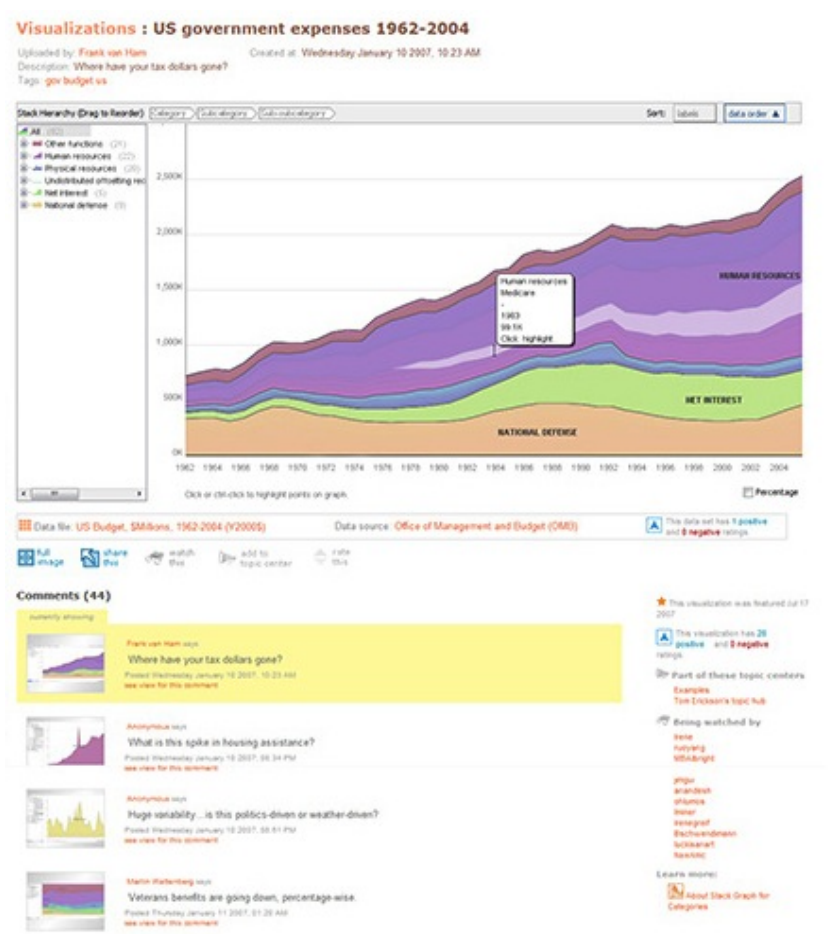

Figure 4: The ManyEyes web interface enables collaborative visualization of shared data. The main focus goes towards the visualization that users can create, customize and annotate with additional information and remarks. The comments section below captures the opinions of users about the data and the visualization, while at the same time allowing them to create bookmarks of the representation.

\section{Application Scenarios}

In the following subsections we provide five detailed real-world examples of scenarios in which collaborative visualization tools have been used. With this section, we outline the importance of dedicated visualization tools and techniques for specific work scenarios.

\subsection{Collaborative Visualization on the Web: Many Eyes}

Many Eyes ${ }^{17}$ is a social data analysis website where people can upload, visualize, and discuss datasets using a set of pre-defined visual representations and a rich set of tools for annotation, feedback, and mashup. The stated goal of the website is to "democratize" visualization technology by exposing the technology to the broadest possible audience. Because of its web-based design, it is an example of an asynchronous, distributed collaborative visualization tool: collaborators access the website using their browsers through the Internet from different places and at different times.

Many Eyes is a community-participation website, similar in multiple ways to other Web 2.0 sites like YouTube, Flickr, Wikipedia, etc. However, unlike these sites, the purpose of Many Eyes is to support several levels of engagement including simple viewing of the data, interacting with and exploring the data, as well as sharing data (uploading) and creating new visualizations. Accessing the website requires only a standard web browser and a Java runtime environment.

The most important features of Many Eyes are its mechanisms for social sensemaking and collaboration. The textual comment as the main communication mechanism of the website can be added to any visualization and dataset created or uploaded on the site just like a user would comment on a blog post or in an online discussion forum (see Figure 4). However, comments and messages alone are not sufficient for establishing common ground ${ }^{44}$ necessary for efficient collaboration. Many Eyes supports this process with two additional features: bookmarks and annotations. A bookmark is simply a snapshot of the full state of a visualization and can optionally be stored together with a comment. Annotations are also linked to comments, but are used to highlight specific items within the state of a visualization (as opposed to the full state, as for a bookmark). The highlighting is simply done by selecting particular items in a visualization when the comment is added.

Collaboration is also used for structuring the content on Many Eyes. Because uploaded data on the site can come from any area of interest (political, economical, technical, networking, etc), the site also supports grouping datasets and, implicitly, their visualizations into topic centers. Similar to YouTube and other communityparticipation websites, Many Eyes users have the possibility to rate datasets and visualizations. This means that the community also has a quantification role in terms of correctness, as avoiding inaccurate conclusions from faulty data or representations is highly desired. It 
The final, definitive version of this article is published in Information Visualization, 10(4):310-326, October/2011 by SAGE Publications Ltd, All rights reserved. (c) [The Author(s)]

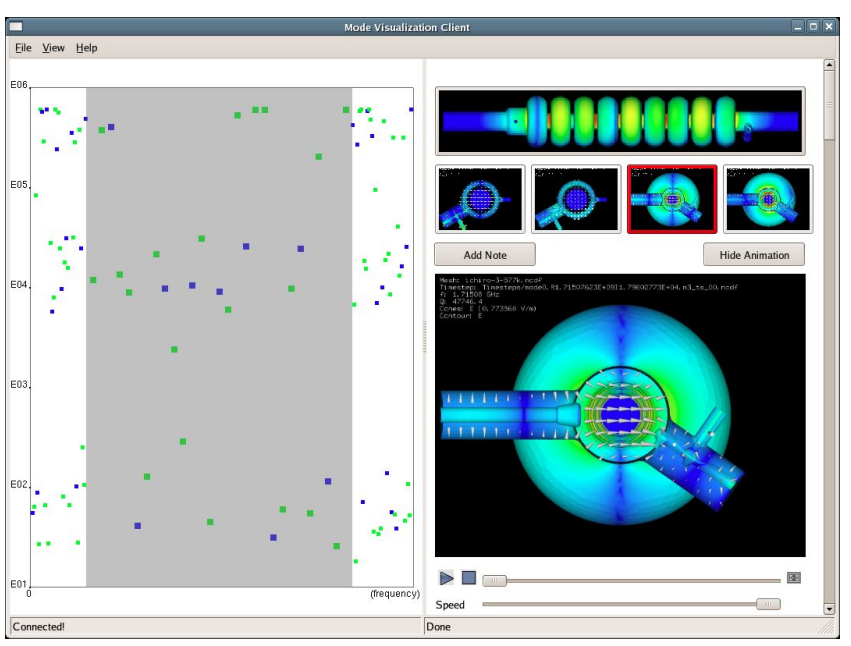

Figure 5: An interface for visualizing accelerator simulation results shared by scientists in an internationally collaborative project. ${ }^{46}$ Each point in the left half of the window represents a specific simulation run. By selecting a point, the user sees corresponding data in the form of images and animations as well as notes made by other users in the right half of the window

also adds to user identity and helps in reputation formation, which is vital both for building a community as well as providing social-psychological incentives to participants. ${ }^{45}$

Finally, Many Eyes supports not only on-site but also off-site communication. This means that the registered users may collaborate not only directly on the Many Eyes website, but also have the ability to bring their visualizations, analysis and insights to their own online communities (e.g., social networks, forums, blogs) by embedding a visualization or a dataset in that context. In fact, in later work, the Many Eyes designers found that rather than the site becoming an online community in its own right, it had evolved to become a "community component" as part of the larger Web 2.0 ecosystem, and that this was enabled by visualizations on the site being easily embeddable into other communities.

\subsection{Collaborative Visualization for Scientific Research}

Many major science investigations such as high energy physics, computational chemistry, climate modeling, and astronomical studies are generating massive amounts of data that are stored in central or distributed storage repositories for sharing. Each such investigation typically involves a large number of scientists, analysts, and students, who utilize the data in their respective studies in a collaborative manner. The notion of "collaboratory" 47 was introduced to support such largescale investigations. In particular, with the flourishing of the Internet, many web-based collaboratories have been established and put into operation for many major science areas. Some of the well-known ones include early projects such as the Upper Atmospheric Research Collaboratory (UARC) ${ }^{48}$ and TeleMed, ${ }^{49}$ and more recent ones include the Particle Physics Data Grid Collaborative Pilot, ${ }^{50}$ the Earth System Grid, ${ }^{51}$ National Fusion Collaboratory, ${ }^{52}$ and Collaboratory for Multi-Scale Chemical Science. ${ }^{53}$

A collaboratory is more than a data warehouse. It should support the very nature of collaboration in the scientific context where collaboration is driven by the need to share both data and knowledge about the data.

Let us consider the International Linear Collider (ILC) project ${ }^{54}$ which involves researchers from SLAC (Stanford Linear Accelerator Center) in the United States, KEK (High Energy Accelerator Research Organization) in Japan, DESY (Deutsches Elektronen Synchrotron) in Germany, and various U.S. national laboratories. Scientists on this project may run the same simulation code with different parameter settings but do not only look at the output data they generated themselves but also those generated by others. When they examine the data, they can create visualizations and add notes summarizing their findings. Figure 5 shows an example of a collaborative interface used by scientists. Such an analysis activity further grows the repositories with derived data, images, and notes, which must be organized for convenient browsing and comparative analysis. Visualization techniques running inside a web-based interface have been created to display the results of each simulation run in terms of visualization and animations, along with notes made by those who have examined the simulation results. ${ }^{55,56}$

Arguably, the primary goal of an online collaboratory is to focus the collective efforts of the group in order to produce significant and useful results. Yet the path to understanding might be just as valuable as the end results, especially if it can assist the discovery process for subsequent tasks or other endeavors entirely. Capturing and visually analyzing the discovery process has been studied for the task of visualization. ${ }^{57}$

Shared data is useful only if sufficient context about the data is given so that collaborators may understand and apply it appropriately. Therefore, it is important to know how a piece of data relates to the overall data space, user space, and application space. The interactions among collaborators are as valuable as the data itself. By focusing on the dynamics of information exchange, Henline ${ }^{58}$ argues that the key challenges in creating a collaboratory may be social rather than technical. Cogburn ${ }^{59}$ also points out that a collaboratory is a new networked, organizational form that also includes social processes. The communication among collaborators can thus be large and complex, easily becoming difficult to comprehend. Extending the collaboratory concept to include both social and behavioral research could provide opportunities of learning more about the social infrastructure that supports a distributed knowledge network. 
In summary, leveraging visual means and visualization techniques can help collaboratory users discover complex relationships and interactions hidden in the collaborative space, facilitate communication and interaction for a better utilization of aggregated software, hardware, and human resources, and ultimately propel knowledge discovery.

\subsection{Collaborative Visualization for Command and Control: Command Post of the Future}

The Command Post of the Future ${ }^{60}$ (CPOF) was a Defense Advanced Research Project Agency (DARPA) project started in 1997. CPOF is a computer system whose goal was to improve command and control using networked information visualization systems to double the speed and quality of command decisions.

CPOF replaced the fixed command post with a virtual, mobile command post. In collaboration terms this was a same time, different location collaboration. That is, commanders can collaborate with others in the field using a shared workspace to gain real-time situation awareness using both text and graphical representations created by fellow commanders and operations officers. In addition, CPOF allows fellow commanders to view the overall commander's intent and to visualize different courses of action.

CPOF supports commanders with three key capabilities: (a) graphical views: $2 \mathrm{D}$ and $3 \mathrm{D}$ information visualizations, (b) information liquidity: drag-and-drop information analysis across different visualization products; and (c) topsight: visibility of evolving understanding among distributed subordinates and team members. These three areas are integrated in a single system to enable the commander and his or her staff to see information, interact with it (to understand it and create new information), and to selectively and dynamically share their evolving understanding of it for analysis, planning, and execution.

$\mathrm{CPOF}$ is able to dynamically incorporate new information which can be from data feeds or from user-entered data. The visualizations in CPOF work on live data and are continually updated to reflect changes in the data. Users have both private and public spaces. A user can work with live data in a private space and drag and drop the analysis into a public space or work product. Users share data but also can tailor their visualizations to capture the way they think.

Figure 6 shows a user's private space and a workspace that a number of users might share. Using this mapbased visualization, distributed commanders can create a plan using information from the local regions. Other visualizations have been built on top of schedules, timelines, as well as tabular and hierarchical templates. Users can interact with the shared workspace independently of each other. Using a voice channel as well, users can talk and use gestures on the screen to collaborate. It is also possible to collaborate asynchronously and to move back and forth between the modes.

CPOF provided radical new capabilities for improving decision making by operational commanders, providing dynamic tailored visualization and deep collaboration tools for improved situation awareness and course-ofaction development and dissemination. The use of visualizations allows field operations officers to transfer situation awareness without a text explanation. This information can be viewed in different ways by the individuals consuming the information. As consumers can view the information in their preferred display, explanations are eliminated, the situation awareness for the commander involved is increased, and the time required for decisions to be made is significantly reduced. The operational tempo is increased because of faster recognition and better understanding of significant battlefield changes, faster and more complete exploration of available courses of action, and more rapid and accurate dissemination of commands. A smaller and more mobile command structure requires fewer staff members, reduced deployment requirements, and a more distributed command organization with an increased span of control. CPOF is composed of modular components which can be scaled and tailored to fit different command environments. The early Army assessment verified that CPOF improved situational awareness, decreased time required for decision making, and increased clarity in information. ${ }^{61} \mathrm{CPOF}$ was successfully transferred from DARPA research to the Department of the Army in 2006.

CPOF is an example of a successful transition of a research program into an operational part of the US Army. CPOF is also an example of how end-user input and feedback were used in shaping the capabilities of the program and providing a robust, battlefield hardened, useful product.

\subsection{Collaborative Visualization for Environmental Planning}

Collaborative visualization in environmental planning has benefited from the confluence of two bodies of scientific literature that have rapidly emerged during the last two decades-Information Visualization and Collaborative Knowledge Construction. ${ }^{64}$ Collaborative knowledge construction is a branch of decision science that deals with multi-party decision-making with the help of communication and visualization tools. ${ }^{62,65}$ These tools often allow users to engage in asynchronous communication and offer intuitive graphical user interfaces (GUIs) for interaction with the data and models. ${ }^{62,63,65-67}$ The use of computer-based visualization tools has become an integral component of collabora- 


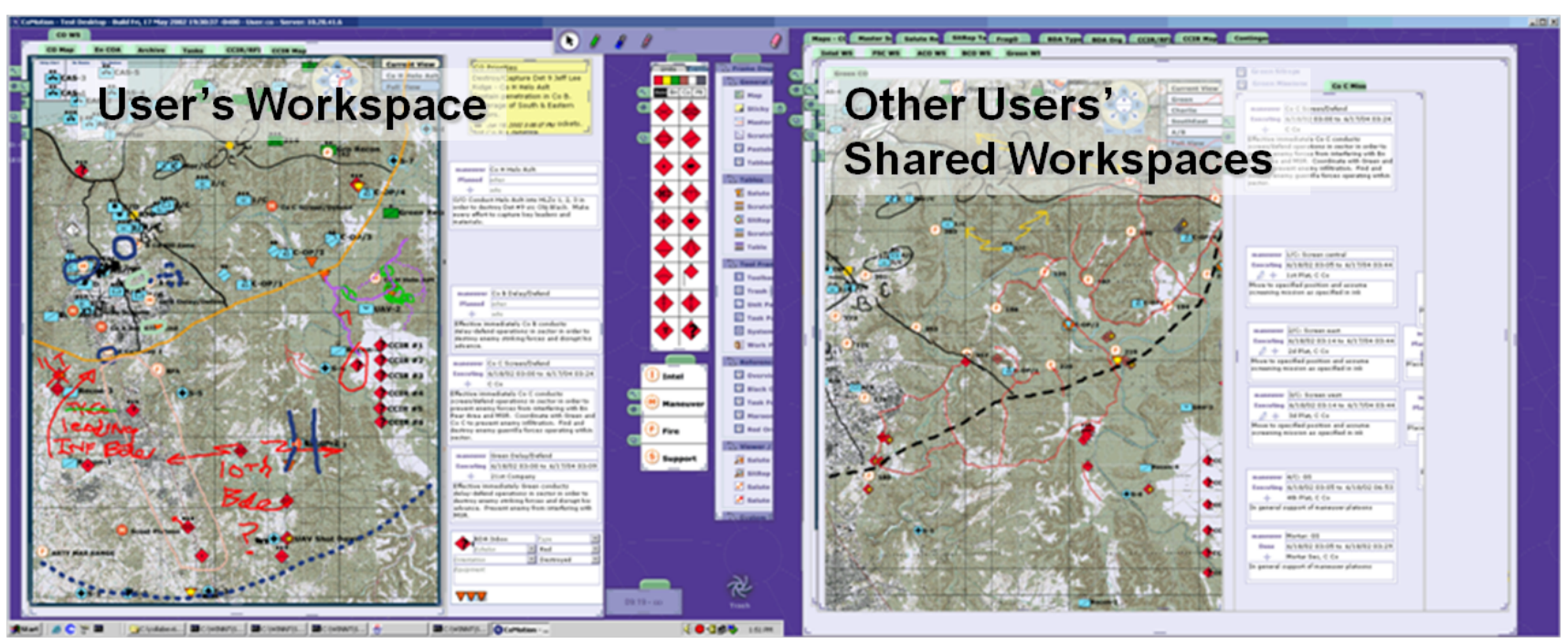

Figure 6: Command Post of the Future with a private and shared workspace (image (c) General Dynamics Corporation).

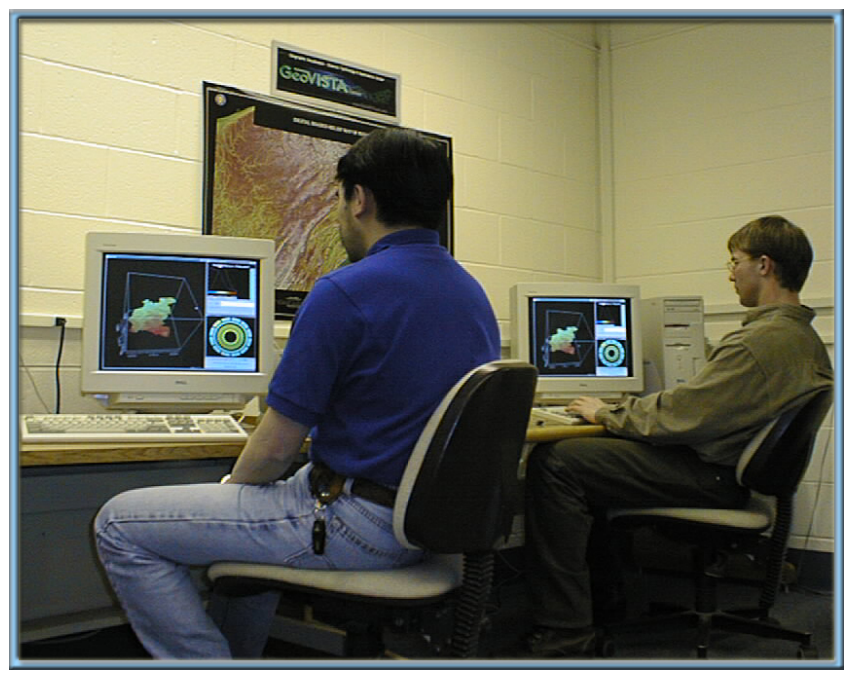

Figure 7: The collaborative geovisualization tool by MacEachren and Brewer ${ }^{62,63}$ showing two linked desktops that supported real-time collaboration with the application (figure courtesy of Alan MacEachren).

tive knowledge construction and its strong links to the field of information visualization is long overdue.

Environmental planning involves collaborative problem solving through effective communication of options, persuasion, plan making, monitoring, and often political strategizing to enable communities to achieve their desired futures. ${ }^{68}$ Hence such planning usually involves multiple stakeholders in the problem formulation and the design of plans. In other words, environmental planning is inherently a political process. The use of collaborative visualization tools helps in defining the problem through multiple perspectives and provides a common platform for examining the consequences of various actions through time and space. Computersupported collaborative visualization in environmental planning provides decision-makers the ability to (1) distill knowledge through mining large multidimensional data sets, (2) run models and simulations to explore the consequences of particular actions, (3) communicate results, scenarios, and opinions to other stakeholders, and (4) discuss, debate, and develop support for specific courses of action.

An example for a collaborative visualization tool for environmental planning is the synchronous and distributed collaborative geovisualization environment proposed by Brewer et al. ${ }^{62,63}$ and shown in Figure 7. The tool enables the exploration of climatic time series via interactions and animations, while offering support for collaborative sensemaking. Users can interact and change the 3D rendering of terrain-dependent temperature and precipitation in order to gain insight. Furthermore, multivariate climatic data can be simultaneously manipulated by multiple users positioned at different locations. The color scheme of the application changes during the interactions of the users to highlight the actions they are performing, such that remote users know how exactly the previous view has changed. The geovisualization system uses a centralized architecture that synchronizes all client views at each major interaction of a particular user, allowing the other users to work on and see the same 3D representation at a moment in time. Other examples of the use of collaborative visualization tools can be found in the Decision Theater at Arizona State University. ${ }^{69}$ One such example involves the use of the Solar Market Analysis and Research Tool that integrates disparate data related to deployment of solar power generation facilities in Arizona. The tool has allowed public-private engagement through unique visualization capabilities and interactive data manipulation and analysis. This has enabled a comprehensive vision of solar potential in Arizona through specific options for planning and locating solar facilities in a costeffective manner. 


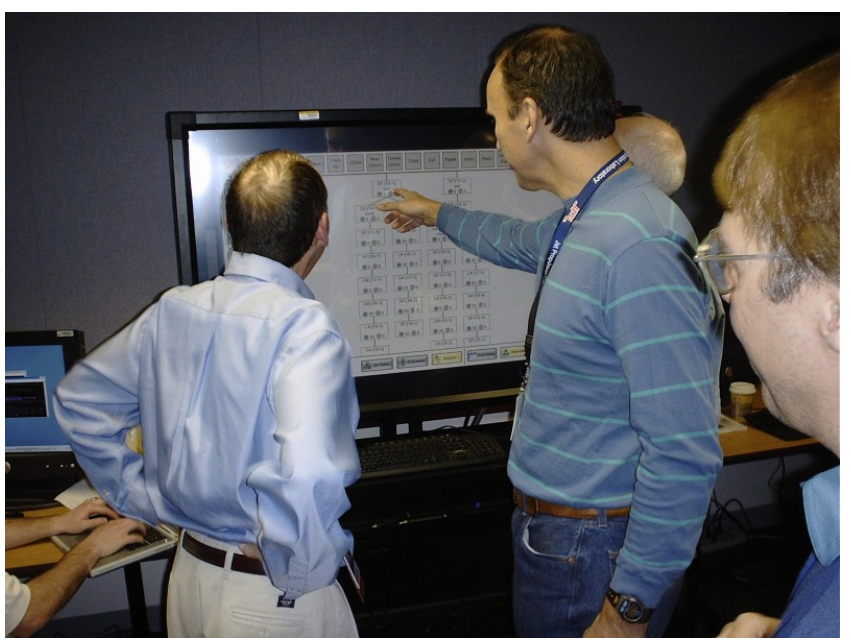

Figure 8: Scientists using the SOLTree application at NASA (image (C)Jay Trimble).

\subsection{Collaborative Visualization for Mission Planning}

The SOLTree application, a part of the MERBoard ${ }^{70}$ platform, is an example of a successful co-located collaborative visualization system. The MERBoard platform was built as a collaborative workspace for colocated scientists for NASA's Mars Exploration Rovers (MER) mission. The technology uses several large multi-user displays, 18 of which were integrated into the work environment of the NASA Jet Propulsion Lab. MER missions required a high degree of collaboration and coordination between teams of engineers and scientists as well as within these teams themselves. ${ }^{71}$

The SolTree tool (see Figure 8) was intended for teams of scientists to create visual tree structures that represented possible next actions for the Mars Rovers. Plans were represented by the nodes, paths, and branches of the tree structure together with annotations of these plans. The trees that were collaboratively created with the tool were used to track progress but also to later present to the larger work group to facilitate discussion about long-term planning. The tool was the most actively used MERBoard application at the beginning of the mission. Collaborative work around SOLTree was typically conducted by small groups of three to 12 people, but the numbers decreased as the mission progressed. While collaborative authoring occurred, it also often happened that an individual would draft a plan alone and later gather other scientists around the display to discuss the plan and receive feedback. While the display and tool were successful for small groups, the scientists had to transfer the results of their planning to larger screens to discuss plans with the larger work group.

The visualization itself was simple but powerful for the scientists to provide a graphical overview of options and alternatives already discussed, to provide a brainstorm- ing structure, and to force annotation of considered alternatives. As such the application with its large-display setting in the workspace also served the purpose of a persistent information display providing community awareness. ${ }^{71}$ It was, therefore, not only a co-located synchronous collaboration tool but also helped asynchronous collaboration in that it showed past decisions and evolution of plans to others entering the decision making process. SOLTree is an interesting success story of a fairly simple visual planning tool which despite its simplicity in terms of visualization provided important support for a team of scientists in a highly collaborative, coordinated, and dynamic work environment.

\section{Unique Focus of Collaborative Visualization}

As previously discussed, collaborative visualization lies at the intersection of two major research fields: traditional visualization and computer-supported cooperative work (CSCW). Clearly, both of these fields have long and rich histories, and we must be aware of both of these to make contributions to collaborative visualization. The position of collaborative visualization as a sub-area within two larger research fields brings a specific focus of its own. In the following we point out this unique focus from the standpoint of visualization research. We concentrate on the actual intersection between the fields (summarized in Table 1) to point out the unique challenges and requirements that require attention from researchers.

\subsection{Users and Tasks}

From a core visualization standpoint, the most obvious focus of collaborative visualization is the addition of participants beyond the canonical single analyst that traditional visualization software is designed for. Having multiple participants is what transforms the analytical sensemaking ${ }^{12}$ process into a collaborative one and gives rise to all of the challenges discussed here.

Similar to the broad range of CSCW research, collaborative visualization research has explored a range of group sizes starting from the basic paired-analysis scenarios $^{39,72}$ to internet-sized audiences. ${ }^{73}$ The focus in collaborative visualization, however, is on a specific type of audience with either specific analysis questions and interests or even specific data-related background knowledge ("expert users"). This specific audience also carries specific tasks centered around visual representations and presentations of data ranging from specific work and/or domain-related data analysis questions to more open data exploration in museums or online. ${ }^{2}$ 
The final, definitive version of this article is published in Information Visualization, 10(4):310-326, October/2011 by SAGE Publications Ltd, All rights reserved. (c) [The Author(s)]

\begin{tabular}{ll}
\hline Aspect & Specific Collaborative Visualization Challenges \\
\hline users & multiple participants \\
tasks & collaborative activity-centric \\
cognition & collaborative foraging \& collaborative sensemaking \\
results & consensus, shared insight \\
interaction & multiple inputs \\
visual representations & multiple displays, novel display and input technology \\
evaluation & social interaction \\
\hline
\end{tabular}

Table 1: Specific challenges to address in the research space intersecting collaborative work and visualization.

\subsection{Cognition and Results}

One of the main differentiating factors from the wider field of CSCW research is that the focus of collaborative visualization is often not the creation of a "product" (e. g., a photo layout or a text document) but an increased understanding or insight into a dataset, a consensus, or the ability to make informed decisions. Accordingly, collaborative visualization involves unique cognitive activities, such as information foraging and sensemaking. ${ }^{20}$ With this focus, the design of collaborative visualization systems poses challenges in addition to those encountered during the design of visualization systems that are intended to be used by a single person.

In a group setting, the use of collaborative technology needs to support a process of social interaction around the data. This social interaction can have different goals. For example, one may be for the group to arrive at a common understanding of the data through a process of collaborative interpretation, analysis, discussion, and interaction. Another may be to simply enhance ones own learning or knowledge construction by making use of others' interactions with the data. A single person typically works with an information display through a process of viewing and possibly interacting with a visualization, forming a mental model by interpreting the representation, and ideally gaining an insight and forming a decision. ${ }^{74}$ When two people join together in collaborative analysis, they can also both gain individual insights by looking at and interpreting the visualization. However, through social interaction (e.g., discussion and negotiation) they can also build on each others' insights and potentially reach a common understanding of the dataset in order to make informed decisions as a group, derive common recommendations, or take next step actions together after the analysis. In our definition from section 2 we emphasize this contribution to joint information processing activities.

\subsection{Interaction and Visual Representations}

One of the main challenges of visualization research is that data analysis is often a complex task: multistaged, poorly understood, and characterized by dynamic and conflicting information. This in turn means that analysis of data is also often a long-term task or process. Therefore, at least some collaborative visualization tools need to support long-term use by various people with various data-related backgrounds, and varying strategies, goals, approaches, ${ }^{19}$ and temporal access patterns.

In addition, within the process of data analysis, collaborative work can and does occur at different stages: information acquisition, representation and presentation, analysis and interpretation, sharing of analysis results, and making decisions and taking actions. So far, research on collaborative visualization is most often targeted at one of these stages and each has its unique challenges which need to be considered.

More specifically, in contrast to much of general CSCW research where there typically is a single visual representation of an information artifact, visualization focuses on underlying datasets with different representation possibilities and views of the data. These representations are also typically interactive with their own unique view-specific operations that go beyond 2D spatial movement of items in a workspace. For example, researchers have considered the collaborative aspects of multiple-view coordination in co-located settings ${ }^{37}$ or interactive data annotation and view-dependent commenting online. ${ }^{73}$

Another research venue in collaborative visualization has been to find out how existing visual representations and interaction techniques need to be enriched and augmented to better support collaborative settings. ${ }^{41,42,75}$ For example, collaborative brushing and linking using meta-visualizations was introduced to help collaborators in staying aware of each others' actions ${ }^{36}$ and studies have been carried out to see which visualizations were particularly helpful for viewing at different orientations around a tabletop display. ${ }^{76}$ The co-located synchronous quadrant of the time-space matrix, in particular, comes with inherent interaction challenges that arise when multiple people have the possibility to synchronously interact. These challenges are of both social and technical nature. Examples of social interaction challenges involve how to design systems to avoid interaction conflicts, how to work around social norms and conventions (e.g. accidental touching of others' arms or hand), or interaction fatigue. Technical challenges involve, for example how to design multi-person multitouch gestures for data visualization, or how to deal 
with the complex issue of collaborative undo of data interactions or the maintenance of a data interaction history.

Finally, whereas an objective within CSCW has long been exploiting novel computing hardware to support multiple inputs and output that facilitate collaboration, it is only recently that visualization research took the leap beyond the standard mouse-and-keyboard desktop computer. On the other hand, visualization, with its background in computer graphics and emphasis on large datasets, brings a focus on high-performance graphics rendering that is not nearly as common within CSCW research.

\subsection{Evaluation}

The success of visual data analysis is strongly connected to the mental model that a person forms about the data by viewing the visualization. ${ }^{74}$ However, our understanding of how this mental model formation works is still very limited and we know even less about how a group forms an understanding or insight of a dataset. The goal of using a collaborative information visualization system is typically to provide the group with an environment that enriches their data analysis activities beyond what they could come up with as separate individuals. A collaborative data analysis scenario should, therefore, support group insight formation. However, measuring group insight (or even individual insight as pointed out previously ${ }^{77}$ ) is difficult. Similar to the problems inherent in evaluating single-user visualizations, we do not have a clear idea about how to evaluate the possible additional insights or the group learning effect that can be achieved by using such a system. How do you capture group insight or learning? Is the group even important for the construction of insight in the individual? If so, how do we find out?

Stahl ${ }^{13}$ proposes to observe team members' conversations about data discoveries. Where do they agree or disagree, augment or confirm each other? The advantage of observing collaborative formation of insight vs. insight made by a single person is that group members may have to make these processes visible to each other, thereby making them visible to the observer as well. As more collaborative systems are built for data analysis, different methods will have to be tried to evaluate each of these systems.

\section{Collaborative Visualization Challenges and Research Agenda}

One of the main goals of research in collaborative visualization is to enable people to collaboratively use visual representations of data to gain additional understanding, knowledge, and insight into the data-different or more encompassing - than would have been possible had they explored the data individually. To learn more about how this goal can be reached, researchers have to address both the technical challenges of designing and implementing digital and physical environments that support collaborative data analysis, as well as the social aspects of group work.

This section attempts to summarize a number of immediate goals and our own vision of the most urgent and promising directions and goals for collaborative visualization.

\subsection{Address Dedicated Research Challenges}

In order for collaborative visualization systems to become used and adopted, it is important to solve interaction and representation challenges on all areas outlined in Section 5. Interactions with collaborative systems as well as the visual representations that are offered to a team are central to the abilities of each member to work with others, receive related information, and spontaneously react to emerging information and ideas from others. We need to learn more about how to design interactions and representations to specifically support collaborative reasoning and sensemaking.

In the future, we expect data analysis to be conducted as a continuous process that bridges individual and collaborative work. Only when collaborations are quick to set up, do not require considerable overhead to organize, and when the results of a collaboration can be quickly used to inform further work, will these setups be useful in practice. Research on this challenge involves making interactions with collaborative visualizations transparent so that these tools can be used on the fly, spontaneously, and without setup overhead. We therefore echo the recent call for more dedicated research on interaction with visualization systems ${ }^{1}-$ particularly focusing on collaborative interactions and data exchanges. We hope to see more fundamental social and technical collaborative visualization challenges being addressed in the next five years.

\subsection{Engage New Audiences}

It is important for the research community to connect to a wide audience of people with collaborative analysis needs, to study these needs in depth, and to publish the results of an analysis of their needs, requirements, and challenges. As discussed early in the paper, a number of different analysis scenarios, needs, questions, goals, and challenges exist. To arrive at a more encompassing understanding and generalizable overview of collaborative visualization requirements and best practices, we need to ground our understanding in specific real-world examples. Within the next five years we therefore urge researchers to help in establishing connections to a wide 
audience with collaboration needs, to study their collaborative needs and requirements, and publish reports on these investigations.

\subsection{Standardize Collaboration Support}

In the future we expect information visualization systems to become more ingrained in people's every day work processes at a number of different stages of data analysis-from data collection to dissemination of analysis results. At any of these stages, collaboration may be essential to ensure quality decisions, more encompassing solutions, or the integration of different viewpoints. Therefore, it seems reasonable to consider integrating collaboration support as a standard in future visualization systems from the beginning. Retrofitting visualization systems is possible ${ }^{75}$ but not always easy in retrospect, so providing collaborative support from the inception of a new tool will become increasingly important. One way this could be achieved is to develop visualization toolkits which allow developers to easily offer collaboration features in their visualization tools, for example through multiple synchronous inputs for shared displays or dedicated networking capabilities. ProtoVis ${ }^{78}$ and the InfoVis Toolkit ${ }^{75}$ are examples of toolkits where such an integration has begun. Within the next five years we hope to see this integration become a standard.

\subsection{Expand to New Collaborative Spaces}

Collaboration can also occur outside and across the confines of the time-space matrix. ${ }^{5}$ In order to cover collaborative data analysis needs more broadly, we need to expand also to more research on hybrid collaboration scenarios. One example of such a hybrid scenario is mixed-presence collaboration in which collaborative analysis occurs in a shared co-located and distributed setting. For example, Kim et al. ${ }^{79}$ developed a toolkit called Hugin that supports collaborative analysis both on shared tabletop displays as well as across distance on remotely connected tabletop displays. Other hybrid scenarios (e. g., using the same space but used both synchronously and asynchronously) still need further research attention. Within the next five years we hope to see collaborative visualization research to have explored and addressed challenges of a number of different collaboration scenarios.

\subsection{Develop Dedicated Evaluation Methods}

Developing dedicated methods for evaluating visualization systems has been an active topic of research in the last couple of years, and it is clear that assessing the value of visualization to people's work processing, learning, or understanding of a topic is difficult. Similarly, the field of CSCW has been discussing how to evaluate groupware systems for a number of years and a variety of approaches have been proposed-yet never with a focus on assessing systems targeted towards data analysis with visualizations. The challenges have already been outlined above in Section 5.4. Within the next five years, we need to begin to develop new methods to assess the impact of collaborative tools and continue to refine and assess their value across different types of collaborative settings, data, tasks, or group sizes.

\subsection{Integration and Adoption}

Distributed visualization systems ${ }^{17}$ have so far made huge progress towards integration and adoption of data analysis environments in collaborative settings. Several commercial systems have also begun to offer collaborative features, including Tableau Public, Spotfire Decision Site Posters, ${ }^{80}$ or MayaVis. ${ }^{81}$ Within the next ten years we hope to see collaborative data analysis systems become generally more integrated and adopted in a variety of everyday environments and using a variety of different access possibilities (e. g. smartphones, tablets, or large display technology). Collaborative visualization has applicability to a large variety of audiences, from those with a broad range of backgrounds, loose connections, and varying goals to very specific taskoriented work teams. Similarly, these audiences may be dealing with very different data characteristics. For example, collaborative visualization for scientific research is targeted towards a very specific audience and often uses visualization with a high information density. This stands in contrast to collaborative visualization for museum exhibits or shopping windows for broad audiences and data of an often lower information density.

We hope that within the next ten years, research will have moved into practice in new venues and have shown to be successful, important, and enriching in a variety of situations. For researchers it will be important to document efforts of integration and the success of adoption of collaborative visualization in a variety of areas so that the community can benefit more generally.

\subsection{Derive A Higher-Level Understanding}

One of the long-term goals of the community should be to derive a higher-level understanding of collaborative visualization challenges and requirements. In this article we have outlined a number of them, but dedicated research in additional application areas will inevitably broaden our understanding and extend this initial set. As a community we need to encourage research on collaborative visualization, get students and young researchers interested in the topic, and continue 
to publish research from a number of different application areas. We need to learn about new and extended social as well as technical challenges to finally arrive at a higher-level understanding of characteristics of collaborative visualization which may span across different areas. One particularly fundamental challenge is to map out a better understanding of collaborative data analysis as a process, and thus it will be important to specifically study and document how particular audiences conduct data analysis with visualizations collaboratively, what the goals and outcomes of the collaboration are, and how groups reason and how information and knowledge formation are affected by visualization use.

\section{Conclusion}

The future will see collaboration with digital information become a central aspect of people's use of computing technology. The types of social exchanges around digital information can range from very casual online conversations with friends or family members about their social network, to discussions around museum exhibits, planned data explorations in research labs, to decision-making scenarios in conference rooms, or to internet-sized data explorations, discussions, and interpretations. Visualization of data will be central to the many collaborative interactions with digital information given its power in providing quick visual access to data and making information readily understandable. In order to enable and capitalize on this trend, it is important for visualization researchers to find out how we can make collaboration support a standard for data analysis environments.

In this article, we have given a broad overview of collaborative visualization, have highlighted five of its many application scenarios, and provided an overview of the unique focus of collaborative visualization as it is embedded within the broader fields of visualization and computer-supported cooperative work. We used these discussions to derive a set of challenges and a research agenda for the future of collaborative visualization. The article is meant as an inspiration to others to begin or to extend their investigations into collaborative visualization. Numerous open research problems exist and in order for visualization to reach new audiences with our tools, solving these challenges will be essential.

\section{References}

[1] J.J. Thomas, K.A. Cook, editors. Illuminating the Path: The Research and Development Agenda for Visual Analytics. IEEE Computer Society; 2005.

[2] P. Isenberg, U. Hinrichs, M. Hancock, S. Carpendale. Digital Tables for Collaborative Information Exploration. In: C. MuellerTomfelde, editor. Tabletops-Horizontal Interactive Displays.
Human-Computer Interaction Series. Springer Verlag; 2010. p. 387-406.

[3] Z. Pousman, J.T. Stasko, M. Mateas. Casual Information Visualization: Depictions of Data in Everyday Life. IEEE Transactions on Visualization and Computer Graphics. 2007 Nov/Dec;13(6):1145-1152.

[4] R.M. Baecker. Readings in Groupware and Computer-Supported Cooperative Work. San Francisco: Morgan Kaufmann Publishers; 1993.

[5] A.J. Dix, J.E. Finlay, G.D. Abowd, R. Beale. Human-Computer Interaction. London: Prentice Hall; 1998.

[6] R.R. Raje, M. Boyles, S. Fang. CEV: Collaborative Environment for Visualization using Java RMI. Concurrency - Practice and Experience. 1998;10(11-13):1079-1085.

[7] J. Heer, F. van Ham, S. Carpendale, C. Weaver, P. Isenberg. Creation and Collaboration: Engaging New Audiences for Information Visualization. In: A. Kerren, J.T. Stasko, J.-D. Fekete, C. North, editors. Information Visualization-Human-Centered Issues and Perspectives. vol. 4950 of LNCS State-of-the-Art Survey. Springer Verlag; 2008. p. 92-133.

[8] G. Johnson. Collaborative Visualization 101. ACM SiggraphComputer Graphics. 1998 May;32(2):8-11.

[9] L.W.F. Li, F.W.B. Li, R.W.H. Lau. A Trajectory-Preserving Synchronization Method for Collaborative Visualization. IEEE Transactions of Visualization and Computer Graphics. 2006;12(5):989-996.

[10] W. Krüger, B. Fröhlich. The Responsive Workbench. IEEE Computer Graphics and Applications. 1994 May;14(3):12-15.

[11] M. Wattenberg. Baby Names, Visualization, and Social Data Analysis. In: Proceedings of the Symposium on Information Visualization (InfoVis). Los Alamitos, CA, USA: IEEE; 2005. p. $1-7$.

[12] S. Card, J.D. Mackinlay, B. Shneiderman, editors. Readings In Information Visualization: Using Vision To Think. San Francisco, CA, USA: Morgan Kaufmann Publishers; 1999.

[13] G. Stahl. Group Cognition. MIT Press; 2006.

[14] E. Hutchins. Cognition in the Wild. MIT Press; 1996.

[15] E. Wenger. Communities of Practice: Learning, Meaning, and Identity. Cambridge University Press; 1999.

[16] R.N. Zambrano, Y. Engelhardt. Diagrams for the Masses: Raising Public Awareness-From Neurath to Gapminder and Google Earth. In: Diagrammatic Representation and Inference. vol. 5223/2008. Berlin / Heidelberg: Springer-Verlag; 2008. p. 282292.

[17] F.B. Viégas, M. Wattenberg, F. van Ham, J. Kriss, M. McKeon. Many Eyes: A Site for Visualization at Internet Scale. IEEE Transactions on Visualization and Computer Graphics. 2007 Nov/Dec;12(5):1121-1128.

[18] I.J. Grimstead, D.W. Walker, N.J. Avis. Collaborative Visualization: A Review and Taxonomy. In: Proceedings of the Symposium on Distributed Simulation and Real-Time Applications (DS-RT). Los Alamitos, CA, USA: IEEE; 2005. p. 61-69.

[19] P. Isenberg, A. Tang, S. Carpendale. An Exploratory Study of Visual Information Analysis. In: Proceedings of the Conference on Human Factors in Computing Systems (CHI). New York, USA: ACM Press; 2008. p. 1217-1226.

[20] D.M. Russell, M.J. Stefik, P. Pirolli, S.K. Card. The Cost Structure of Sensemaking. In: Proceedings of the Conference on Human Factors in Computing Systems (CHI). New York, NY, USA: ACM Press; 1993. p. 269-276. 
[21] C.S. Ang, D.C. Martin, M.D. Doyle. Integrated Control of Distributed Volume Visualization Through the World-Wide-Web. In: Proceedings of the Conference on Visualization (VIS). Los Alamitos, CA, USA: IEEE Computer Society; 1993. p. 13-20.

[22] L. Renambot, B. Jeong, H. Hur, A. Johnson, J. Leigh. Enabling High Resolution Collaborative Visualization in Display Rich Virtual Organizations. Future Generation Computer Systems. 2009 Feb;25(2):161-168.

[23] J. Wood, H. Wright, K. Brodlie. Collaborative Visualization. In: Proceedings of the Conference on Visualization (VIS). Los Alamitos, CA, USA: IEEE; 1997. p. 253-259.

[24] R. Matsukara, K. Koyamada, Y. Tang, Y. Karube, M. Moriya. VizGrid: Collaborative Visualization Environment for Natural Interaction between Remote Researchers. Fujitsu Scientific \& Technical Journal. 2004 Dec;40(2):205-216.

[25] T.J. Jankun-Kelly, O. Kreylos, K.-L. Ma, B. Hamann, K.I. Joy, J. Shalf, et al. Deploying Web-based Visual Exploration Tools to the Grid. IEEE Computer Graphics and Applications. 2003;23(2):40-50.

[26] J. Leigh, A.E. Johnson, M. Brown, D.J. Sandin, T.A. DeFanti. Visualization in Teleimmersive Environments. Computer. 1999;32(12):67-73.

[27] T. Paper. Data360; 2009. http://www.data360.org/ (last accessed: February, 2009). Website.

[28] J. Stewart, B.B. Bederson, A. Druin. Single Display Groupware: A Model for Co-present Collaboration. In: Proceedings of the Conference on Human Factors in Computing Systems (CHI). New York, NY, USA: ACM Press; 1999. p. 286-293.

[29] F. Guimbretire. Fluid Interaction For High Resolution Wall-Size Displays [PhD Thesis]. Stanford University; 2002.

[30] P. Wellner. Interacting with Paper on the DigitalDesk. Communications of the ACM. 1993 July;36(7):87-96.

[31] M.A. Nacenta, D. Pinelle, D. Stuckel, C. Gutwin. The Effects of Interaction Technique on Coordination in Tabletop Groupware. In: C.G. Healey, E. Lank, editors. Proceedings of Graphics Interface (GI). Mississauga, ON, Canada: Canadian Information Processing Society; 2007. p. 191-198.

[32] M. Ringel Morris, K. Ryall, C. Shen, C. Forlines, F. Vernier. Beyond "Social Protocols": Multi-User Coordination Policies for Co-located Groupware. In: Proceedings of the Conference on Computer-Supported Cooperative Work (CSCW). New York, NY, USA: ACM Press; 2004. p. 262-265.

[33] S.D. Scott, M.S.T. Carpendale, K.M. Inkpen. Territoriality in Collaborative Tabletop Workspaces. In: Proceedings of the Conference on Computer-Supported Cooperative Work (CSCW). New York, NY, USA: ACM Press; 2004. p. 294-303.

[34] A. Tang, M. Tory, B. Po, P. Neumann, S. Carpendale. Collaborative Coupling over Tabletop Displays. In: Proceedings of the Conference on Human Factors in Computing Systems (CHI). New York, NY, USA: ACM Press; 2006. p. 1181-1290.

[35] R. Kruger, S. Carpendale, S.D. Scott, S. Greenberg. Roles of Orientation in Tabletop Collaboration: Comprehension, Coordination and Communication. Journal of Computer Supported Collaborative Work. 2004;13(5-6):501-537.

[36] P. Isenberg, D. Fisher. Collaborative Brushing and Linking for Co-located Visual Analytics of Document Collections. Computer Graphics Forum (Proceedings of EuroVis). 2009 Juni;28(3):1031-1038.

[37] M. Tobiasz, P. Isenberg, S. Carpendale. Lark: Coordinating Co-located Collaboration with Information Visualization. IEEE Transactions on Visualization and Computer Graphics. 2009 Nov/Dec;15(6):1065-1072.
[38] C. Forlines, C. Shen. DTLens: Multi-user Tabletop Spatial Data Exploration. In: Proceedings of the Symposium on User Interface Software and Technology (UIST). New York, USA: ACM Press; 2005. p. 119-122.

[39] P. Isenberg, S. Carpendale. Interactive Tree Comparison for Co-located Collaborative Information Visualization. IEEE Transactions on Visualization and Computer Graphics. 2007 Nov/Dec;13(6):1232-1239.

[40] B. Johnson, A. Fox, T. Winograd. The Interactive Workspaces Project: Experiences with Ubiquitous Computing Rooms. IEEE Pervasive Computing Magazine. 2002 April-June;1(2):67-74.

[41] C. Forlines, R. Lilien. Adapting a Single-User, Single-Display Molecular Visualization Application for Use in a Multi-User, Multi-Display Environment. In: Proceedings of the Conference on Advanced Visual Interfaces (AVI). New York, NY, USA: ACM Press; 2008. p. 367-371.

[42] C. Forlines, A. Esenther, C. Shen, D. Wigdor, K. Ryall. Multi-User, Multi-Display Interaction with a Single-User, Single-Display Geospatial Application. In: Proceedings of the Symposium on User Interface Software and Technology (UIST). New York, NY, USA: ACM Press; 2006. p. 273-276.

[43] D. Wigdor, H. Jiang, C. Forlines, M. Borkin, C. Shen. WeSpace: The Design Development and Deployment of a Walk-up and Share Multi-Surface Visual Collaboration System. In: Proceedings of the Conference on Human Factors in Computing Systems (CHI). New York, NY, USA: ACM Press; 2009. p. 1237-1246.

[44] H.H. Clark, S.E. Brennan. Grounding in Communication. In: R.M. Baecker, editor. Readings in Groupware and ComputerSupported Cooperative Work: Assisting Human-Human Collaboration. San Francisco: Morgan Kaufmann Publishers Inc.; 1993. p. 222-234.

[45] J. Heer, M. Agrawala. Design Considerations for Collaborative Visual Analytics. Information Visualization. 2008;7(1):49-62.

[46] K.-L. Ma, C. Wang. Social-Aware Collaborative Visualization for Large Scientific Projects. In: Proceedings of International Symposium on Collaborative Technologies and Systems. Los Alamitos, CA, USA: IEEE; 2008. p. 190-195.

[47] W.A. Wulf. The Collaboratory Opportunity. Science. 1993 Aug;p. 854-855.

[48] S. Subramanian, G.R. Malan, H.S. Shim, J.H. Lee, P. Knoop, d.T.E. Weymouth, et al. Software Architecture for the UARC Web-Based Collaboratory. IEEE Internet Computing. 1999 Mar;3(2):46-54.

[49] D.G. Kilman, D.W. Forslund. An International Collaboratory Based on Virtual Patient Records. Communications of the ACM. 1997 Aug;40(8):110-117.

[50] U. S. Department of Energy Collaboratories. Particle Physics Data Grid Collaboratory Pilot;. Available from: http://www. doecollaboratory. org/research2/ppdg/homepage.html.

[51] D. Bernholdt, S. Bharathi, D. Brown, K. Chanchio, M. Chen, A. Chervenak, et al. The Earth System Grid: Supporting the Next Generation of Climate Modeling Research. Proceedings of the IEEE. 2005 Mar;93(3):485 -495.

[52] D.P. Schissel, J.R. Burruss, A. Finkelstein, S.M. Flanagan, I.T. Foster, T.W. Fredian, et al. Building the US National Fusion Grid: Results from the National Fusion Collaboratory Project. Fusion Engineering and Design. 2004 Jun;71(1-4):245-250.

[53] Sandia National Laboratories. Collaboratory for Multi-Scale Chemical Science; Last visited: 2010. Website. Available from: http://cmcs.org.

[54] K. Ko. The International Linear Collider. SciDAC Review. 2006;1:17-20 
[55] K.-L. Ma, G. Schussman, B. Wilson, K. Ko, J. Qiang, R. Ryne. Advanced Visualization Technology for Terascale Particle Accelerator Simulations. In: Proceedings of ACM/IEEE Supercomputing Conference. Los Alamitos, CA, USA: IEEE; 2002. p. 1-11.

[56] Y. Wang, J. Shearer, K.-L. Ma. VICA: A Voronoi Interface for Visualizing Collaborative Annotations. In: Proceedings of the International Conference on Cooperative Design, Visualization, and Engineering. Berlin, Heidelberg: Springer-Verlag; 2007. p. 21-32.

[57] K.-L. Ma. Visualizing Visualizations: User Interfaces for Managing and Exploring Scientific Visualization Data. IEEE Computer Graphics and Applications. 2000 Sep/Oct;20(5):16-19.

[58] P. Henline. Eight Collaboratory Summaries. Interactions. 1998 May/Jun;5(3):66-72.

[59] D.L. Cogburn. HCI in the So-Called Developing World: What's in it for Everyone. Interactions. 2003 Mar/Apr;10(2):80-87.

[60] M.C. Chuah, S.F. Roth. Visualizing Common Ground. In: Proceedings of the Conference on Information Visualization (IV). Los Alamitos, CA, USA: IEEE; 2003. p. 365-372.

[61] GlobalSecurity. org. Command Post of the Future [CPOF]; 2010. Accessed 10/19/2010. Available from: http://www. globalsecurity.org/military/systems/ground/cpof.htm.

[62] I. Brewer, A.M. MacEachren, H. Abdo, J. Gundrum, G. Otto. Collaborative Geographic Visualization: Enabling Shared Understanding of Environmental Processes. In: Proceedings of the Symposium on Information Visualization (InfoVis). Los Alamitos, CA, USA: IEEE; 2000. p. 137-141.

[63] A.M. MacEachren, I. Brewer. Developing a Conceptual Framework for Visually-Enabled Geocollaboration. International Journal of Geographical Information Science. 2004 Jan/Feb;18(1):1-34.

[64] I.D. Bishop, E. Lange, editors. Visualization in Landscape and Environmental Planning: Technology and Applications. Taylor \& Francis; 2005

[65] F. Fischer, J. Bruhn, C. Gräsel, H. Mandl. Fostering Collaborative Knowledge Construction with Visualization Tools. Learning and Instruction. 2002;12(2):213-232.

[66] D.L. Schwartz. The Productive Agency that Drives Collaborative Learning. In: Collaborative Learning: Cognitive and Computational Approaches. Oxford, UK: Pergamon/Elsevier Science; 1999. p. 197-218.

[67] M. Baker, T. Hansen, D. Traum. The Role of Grounding in Collaborative Learning Tasks. In: Collaborative Learning: Cognitive and Computational Approaches. Pergamon; 1999. p. 3163.

[68] G. Steinebach, S. Guhathakurta, H. Hagen. Visualizing Sustainable Planning. Springer; 2009.

[69] R.M. Edsall, K.L. Larson. Effectiveness of a Semi-Immersive Virtual Environment in Understanding Human-Environment In- teractions. Cartography and Geographic Information Science. 2009;36(4):367-384.

[70] I. Tollinger, M. McCurdy, A.H. Vera, P. Tollinger. Collaborative Knowledge Management Supporting Mars Mission Scientists. In: Proceedings of the Conference on Computer Supported Cooperative Work (CSCW). New York, NY, USA: ACM; 2004. p. 29-38.

[71] E. Huang, E. Mynatt, J. Trimble. Displays in the Wild: Understanding the Dynamics and Evolution of a Display Ecology. In: Pervasive Computing. vol. 3968 of Lecture Notes in Computer Science. Springer Berlin / Heidelberg; 2006. p. 321-336.

[72] A.C. Robinson. Collaborative Synthesis of Visual Analytic Results. In: Proceedings of the Symposium on Visual Analytics Science and Technology (VAST). Los Alamitos, CA, USA: IEEE; 2008. p. 67-74.

[73] J. Heer, F.B. Viégas, M. Wattenberg. Voyagers and Voyeurs: Supporting Asynchronous Collaborative Information Visualization. In: Proceedings of the Conference on Human Factors in Computing Systems (CHI). New York, NY, USA: ACM; 2007. p. 1029-1038.

[74] R. Spence. Information Visualization. 2nd ed. Harlow, England: Pearson Education Limited; 2007.

[75] P. Isenberg, A. Bezerianos, N. Henry, S. Carpendale, J.D. Fekete. CoCoNutTrix: A Study in Collaborative Retrofitting for Information Visualization. Computer Graphics and Applications: Special Issue on Collaborative Visualization. 2009 Sep/Oct;29(5):44-57.

[76] F. Alallah, D. Jin, P. Irani. OA-Graphs: Orientation Agnostic Graphs for Improving the Legibility of Charts on Horizontal Displays. In: Proceedings of Interactive Tabletops and Surfaces (ITS). New York, NY, USA: ACM; 2010. p. 211-220.

[77] P. Saraiya, C. North, K. Duca. An Insight-Based Methodology for Evaluating Bioinformatics Visualizations. IEEE Transactions on Visualization and Computer Graphics. 2005 Jul/Aug;11(4):443-456.

[78] J. Heer, M. Bostock. Declarative Language Design for Interactive Visualization. Transactions on Visualization and Computer Graphics. 2010 Nov/Dec;16(6):1149-1156.

[79] K. Kim, W. Javed, C. Williams, N. Elmqvist, P. Irani. Hugin: A Framework for Awareness and Coordination in Mixed-Presence Collaborative Information Visualization. In: Proceedings of the Conference on Interactive Tabletops and Surfaces (ITS); 2010. p. 231-240.

[80] Spotfire, Inc. . DecisionSite ${ }^{\circledR} \quad$ Posters; 2007. http://www.spotfire .com/ (accessed March 2007). Website.

[81] MayaViz. CoMotion ${ }^{\circledR} ;$ 2007. http://www.mayaviz. com/http://www.mayaviz.com/ (last accessed March 2007). Website. 